\title{
Saúde e empoderamento das mulheres: estudo de caso do Programa Mais Médicos em municípios com presença de médicos cubanos
}

\author{
Yamila Comes, ${ }^{1}$ Lucélia Luiz Pereira, ${ }^{2}$ Paloma Simas, ${ }^{3}$ Isabela Ribeiro, ${ }^{3}$ Gabriel Vivas ${ }^{4}$ \\ e Carlos Rosales ${ }^{4}$
}

Como citar Comes Y, Pereira LL, Simas P, Ribeiro I, Vivas G, Rosales C. Saúde e empoderamento das mulheres: estudo de caso do Programa Mais Médicos em municípios com presença de médicos cubanos. Rev Panam Salud Publica. 2020;44:e62. https://doi.org/10.26633/RPSP.2020.62

RESUMO

Objetivo. Descrever as representações de ser mulher das usuárias do Programa Mais Médicos (PMM), com perspectiva de gênero e raça, e as mudanças que o PMM trouxe quanto ao empoderamento e cuidado da saúde.

Métodos. Trata-se de um estudo de caso descritivo, de corte transversal. O trabalho de campo foi realizado mediante entrevistas semiestruturadas, aplicação de uma técnica evocativa de associação de palavras e grupos focais em municípios com médicos cubanos, com amostras de tipo nominal para escolha dos municípios e de tipo intencional para a escolha de participantes. $O$ tamanho das amostras foi definido em campo com base na técnica da saturação teórica. Os dados foram analisados por meio de análise de conteúdo e análise prototípica. Resultados. A cobertura da atenção básica foi fortalecida com os aportes do programa, segundo os quatro gestores entrevistados. As mulheres (103 na técnica evocativa e 120 nos grupos focais) relataram mudanças no modelo de atendimento, que se tornou mais humanizado, com impacto sobre sua percepção sobre os serviços de saúde, sobre a consulta médica, sobre os médicos e sobre a imagem de si mesmas e, em menor medida, sobre as práticas de cuidado da saúde.

Conclusões. O PMM trouxe ganhos no empoderamento individual das mulheres, com reflexos potencialmente positivos para os comportamentos em saúde.

Palavras-chave Programas nacionais de saúde; direitos da mulher; assistência integral à saúde; políticas públicas antidiscriminatórias; atenção primária à saúde; Brasil.

No Brasil, o Sistema Único de Saúde (SUS), implementado na década de 1990 a partir do movimento da reforma sanitária no país, se consolidou como política pública de Estado que se fundamenta na concepção de saúde como um direito universal. Nesse contexto, a atenção básica é designada como principal porta de entrada das redes de atenção à saúde (RAS), além de coordenadora/ordenadora dos cuidados e dos serviços disponibilizados pelo SUS. Apesar de ser considerada uma estratégia fundamental para garantir o direito à saúde no Brasil, a atenção básica enfrenta limitações de recursos e dificuldades para incorporar profissionais em todas as equipes, principalmente médicos, o que representa um obstáculo para uma resposta efetiva (1).

Na tentativa de suprir a carência de médicos e ampliar o acesso da população aos serviços de saúde, em 2013 o Governo Federal criou o Programa Mais Médicos (PMM) (2). Entre os principais objetivos do programa, merecem destaque a diminuição da carência de médicos, o fortalecimento da prestação de serviços na atenção básica por meio da incorporação de

\footnotetext{
1 Pesquisadora independente, Buenos Aires, Argentina. $\triangle$ ycomes@gmail.com

2 Universidade de Brasília (UnB), Departamento de Serviço Social, Brasília (DF), Brasil.
}

$\begin{array}{ll}3 & \text { Pesquisadora independente, Brasília (DF), Brasil. } \\ 4 & \text { Organização Pan-Americana da Saúde (OPAS), Brasília (DF), Brasil. }\end{array}$

Este é um artigo de acesso aberto distribuído sob os termos da Licença Creative Commons Attribution-NonCommercial-NoDerivs 3.0 IGO, que permite o uso, distribuição e reprodução em qualquer meio, desde que o trabalho original seja devidamente citado. Não são permitidas modificações ou uso comercial dos artigos. Em qualquer reprodução do artigo, não deve haver nenhuma sugestão de que a OPAS ou o artigo avaliem qualquer organização ou produtos específicos. Não é permitido o uso do logotipo da OPAS. Este aviso deve ser preservado juntamente com o URL original do artigo. 
médicos nas equipes de Estratégia Saúde da Família (ESF), a ampliação da formação em universidades federais e a melhoria da infraestrutura das unidades de saúde (3). Apesar dos desafios para que a atenção básica se transforme em coordenadora das redes (4), há evidências de que esse papel foi facilitado pela implementação do PMM (5).

A concepção ampliada de saúde estabelecida no SUS, a partir do reconhecimento dos determinantes sociais, entende que os serviços devam contemplar aspectos sociais, psicológicos e econômicos, buscando garantir integralidade e intersetorialidade. Além disso, transversalidade e interseccionalidade são categorias fundamentais na implementação de ações que busquem reduzir as iniquidades em saúde vinculadas às questões de gênero e raça. Para Crenshaw (6), a interseccionalidade abarca a complexidade da vivência, considerando a existência dos chamados eixos de subordinação, os quais podem ser referentes ao pertencimento racial, de gênero, à orientação sexual e à condição de classes, entre outros. Esses eixos podem se cruzar, levando a situações de desigualdade que recaem com maior intensidade sobre determinados indivíduos ou grupos. Já a categoria transversalidade diz respeito à necessidade de o Estado incorporar nas políticas públicas as dimensões de gênero e raça com o objetivo de combater as discriminações e as desigualdades sociais e econômicas (7).

O Brasil ainda enfrenta problemas para erradicar as desigualdades de gênero, devido à força dos preconceitos imiscuídos na cultura. Destaca-se a influência histórica do patriarcado sobre as concepções de família, domínio e reificação: além da casa, também a mulher é compreendida como propriedade, principalmente em termos de sexualidade (8).

Desses cenários derivam diversos problemas para o campo da saúde, dada a grande influência das relações de sexo e gênero sobre o bem-estar e o adoecimento das mulheres (9), e também para os serviços de saúde, uma vez que o SUS preconiza o princípio da equidade. É fundamental considerar a presença dos estereótipos de gênero como reforço das práticas de violência contra as mulheres e das concepções fundadas nas desigualdades de gênero. No que tange à violência doméstica, percebe-se um abismo entre a mesma e os serviços de saúde, refletido na baixa procura por atendimentos para a questão dos abusos (10). O apagamento da condição das lésbicas e de suas necessidades também representa ônus ao direito à saúde (11), ferindo os princípios da equidade e da integralidade.

Para construir estratégias de enfrentamento do sexismo, as mulheres precisam se empoderar. $\mathrm{O}$ conceito de empoderamento não tem um caráter universal; existem diferentes perspectivas conceituais, embora a maioria dos autores coincida em definir três níveis de empoderamento, fazendo alusão às esferas individual, relacional ou organizacional e comunitária (12). Gohn (13) define empoderamento como a capacidade dos grupos de poderem decidir sobre questões que lhes são próprias. Os três níveis de empoderamento estariam interrelacionados e circundados pelas relações de poder $(13,14)$. No nível individual, o empoderamento envolve consciência sobre o ganho de poder e autonomia - com ganhos de autoestima, capacidade de decisão e confiança, que são dimensões básicas do empoderamento. No nível relacional entraria o olhar do território, do contexto e das relações de poder onde o indivíduo está inserido. No nível contextual estaria a ação propriamente dita, por meio da participação para mudar a situação desejada. Cabe destacar que as modificações em um nível desencadeiam reações nos outros, podendo essas modificações iniciar-se em algum dos níveis e posteriormente atingir os outros (14).

No caso das mulheres, o empoderamento se relaciona com a necessidade de reverter as desigualdades existentes nas sociedades patriarcais (a falta de oportunidades econômicas, políticas e sociais) e de exigir os direitos que lhes são próprios (como o direito à saúde) (14). O empoderamento das mulheres implica em questionar tal distribuição desigual do poder, sendo um pré-requisito para melhorar os resultados em saúde (15).

Buscando discutir as contribuições do PMM para o empoderamento das mulheres usuárias do SUS, o presente estudo tem como objetivo descrever as representações de ser mulher por parte das usuárias do PMM e as mudanças que o Programa trouxe quanto ao empoderamento e ao cuidado da saúde dessa população.

\section{MATERIAIS E MÉTODOS}

Adotou-se como estratégia metodológica o estudo de casos múltiplos, apropriado para investigar fenômenos contemporâneos em profundidade no seu contexto real (16). O projeto, realizado no contexto da Organização Pan-Americana da Saúde (OPAS), enfocou os médicos cubanos, que representavam, no momento do estudo, em torno de $65 \%$ do total de médicos do Programa.

Os casos analisados são originários de quatro municípios brasileiros das regiões Norte (Rio Branco, estado do Acre), Nordeste (Teixeira de Freitas, Bahia), Sudeste (Itapecerica da Serra, São Paulo) e Centro-Oeste (Gama, Distrito Federal). A população de todos os municípios era maior do que 100 mil habitantes. $\mathrm{O}$ índice de desenvolvimento humano municipal (IDHM) variou de médio a alto $(0,685$ a 0,792$)$, com taxa elevada de população urbana (de 91,8\% a 99\%). Para a escolha dos municípios, partiu-se de uma amostra de casos nominais (17). Para isso, foram consultados todos os representantes médicos do PMM nos estados brasileiros. Esses representantes indicaram municípios onde o programa oferecia práticas exitosas, tendo sido selecionados os quatro municípios do presente estudo.

Foram utilizadas três técnicas de construção de dados: a primeira consistiu em uma entrevista semiestruturada com quatro gestores e nove médicos cubanos do PMM, com o intuito de analisar aspectos relacionados à estrutura do SUS e implantação do PMM. A segunda foi a técnica de evocação livre de palavras (TELP), aplicada a 103 mulheres. A literatura recomenda uma amostra de cerca de 100 pessoas para garantir estabilidade e eliminar a influência de elementos de casos extremos (18). Assim, a TELP foi aplicada a 25 mulheres em Rio Branco e outras 25 em Teixeira de Freitas; no Gama e em Itapecerica da Serra, aceitou-se a solicitação de três mulheres que tiveram interesse em participar, sendo o número de participantes 27 e 26 mulheres, respectivamente, nesses municípios. Cabe esclarecer que, considerando que a população negra também está sujeita a vulnerabilidades na saúde, com racismo estrutural materializado nas organizações a partir do racismo institucional (19), o que desprotege socialmente essa população e provoca iniquidades na atenção à saúde, a equipe trabalhou com as perspectivas de gênero e raça, integrando tais categorias às análises. Portanto, foram investigadas questões de gênero, raça e saúde no conteúdo representacional produzido pelas mulheres, que enfocou: o atendimento na unidade básica de saúde, ter médicos cubanos no município, ser mulher, ser uma mulher negra/branca e ser uma mulher lésbica. 
A terceira técnica foi o grupo focal, realizado com 120 usuárias do PMM, distribuídas em oito grupos (dois grupos por cidade, com 32 participantes no Gama, 22 em Itapecerica da Serra, 36 em Rio Branco e 30 em Teixeira de Freitas), com o intuito de responder questões sobre as percepções e mudanças na saúde, práticas de cuidado e acesso à saúde e empoderamento a partir do PMM. Os grupos foram realizados em unidades básicas de saúde pela manhã e pela tarde, dependendo da disponibilidade das mulheres, e coordenados pela equipe de pesquisa (YC, LLP, PS, IR).

As entrevistas semiestruturadas foram realizadas com os secretários de saúde de cada um dos municípios escolhidos. A entrevista semiestruturada com médicos teve também um critério intencional baseado na disponibilidade do médico cubano para fazer a entrevista. $\mathrm{O}$ tamanho da amostra foi determinado pelo critério de saturação teórica (20).

Para aplicar a técnica evocativa e formar os grupos focais, foi também utilizado o critério da amostra intencional (18). Assim, a amostra foi escolhida em postos de saúde com presença de médicos cubanos e com grande assiduidade de usuários. Nos postos de saúde foi ofertada a participação aos usuários que estavam esperando pela consulta. Os dados construídos a partir da técnica associativa foram analisados com base na teoria das representações sociais, especificamente a análise prototípica (21), e com apoio do software livre Iramuteq (http:// www.iramuteq.org). Os dados levantados nos grupos focais e entrevistas foram analisados a partir da análise de conteúdo (22) e com o auxílio do software Atlas.ti (https://atlasti.com/). Os resultados das técnicas foram integrados por triangulação metodológica (23). O trabalho de campo foi realizado em junho de 2018. Os dados foram gravados em áudio e posteriormente transcritos.

O presente estudo foi aprovado pelo Comitê de Ética em Pesquisa do Centro Universitário de Brasília (CEUB) (protocolo 2.673.593/18). Todos os participantes assinaram um termo de consentimento livre e esclarecido. Foi mantida a confidencialidade dos dados e o anonimato dos participantes, sendo os instrumentos desidentificados.

\section{RESULTADOS}

\section{Entrevista semiestruturada com gestores e médicos}

Os gestores (três mulheres e um homem) e os médicos cubanos do programa (seis mulheres e três homens) descreveram mudanças positivas a partir do PMM, pela maior prioridade dada às ações de prevenção. Conforme as entrevistas, a presença diária dos médicos nas unidades básicas de saúde (UBS) fortaleceu os princípios da longitudinalidade e da integralidade do cuidado. Assim, de acordo com os gestores, o programa trouxe alívio por ser resolutivo e contribuir para o desenvolvimento de um cuidado diferenciado e de fortalecimento do vínculo com a população.

Os médicos cubanos avaliaram de forma positiva a estrutura do SUS. No entanto, a partir da atuação nas equipes das UBS, destacaram a necessidade de melhorar a organização da rede de serviços em relação aos encaminhamentos para as especialidades e contrarreferência e também a ampliação de recursos humanos. Indagados sobre demandas relacionadas à violência, os profissionais afirmaram que atendiam mulheres que sofreram violência, mas que essa não era a queixa principal delas ao buscarem o atendimento.
Sobre a implantação do PMM, os gestores destacaram como mudanças a ampliação do número de atendimentos e da qualidade da atenção. Ao relatar a implantação do PMM no Brasil, os gestores destacaram que, inicialmente, havia preconceito contra os cubanos, sendo que as pessoas associavam a cor dos médicos à (in)competência profissional, duvidando inclusive de que esses médicos tinham formação em medicina.

\section{Vivências de gênero, raça e cuidado da saúde das participantes}

A média de idade das 103 participantes na técnica evocativa foi de 42 anos (19 a 77 anos). Os níveis de escolaridade predominantes foram o ensino médio incompleto e completo respectivamente (tabela 1). As participantes indicaram que não era fácil ser mulher. Enfatizaram a necessidade de serem guerreiras e corajosas para enfrentar as condições de mãe e esposa, trabalhar dentro e/ou fora de casa e ainda se ocupar dos filhos e da saúde de toda a família, além de lidar com os riscos e a vulnerabilidade que o machismo e o patriarcado impõem à mulher com o intuito de dominação (24). Resultados concordantes encontraram-se nas representações sociais sobre o que significa "ser uma mulher" (445 evocações). Nessas representações, as dificuldades foram reforçadas por termos como "guerreira", "sobrecarga" e "trabalhadora". Também foram evocadas palavras relacionadas ao modelo patriarcal, tais como "mãe", "cuidadora" e "carinhosa". A palavra machismo pouco apareceu como conteúdo representacional, o que denota que ainda não existe uma reflexão consolidada sobre a existência de um modelo baseado no patriarcado.

O preconceito manifestado pelas mulheres negras (89 se autodeclararam negras) apareceu em primeiro lugar no núcleo central das representações pela técnica evocativa (330 evocações). O resultado comprova o mito da democracia racial e, ao mesmo tempo, mostra o sofrimento dessas mulheres em pertencerem a esse grupo racial. Sobre ser uma mulher branca, foram evocadas

TABELA 1. Idade e escolaridade das usuárias do Programa Mais Médicos na técnica evocativa livre de palavras em municípios selecionados, Brasil, 2018

\begin{tabular}{lrr}
\multicolumn{1}{c}{ Característica } & No. & $\%$ \\
Idade (anos) & & \\
18 a 29 & 24 & 23,3 \\
30 a 39 & 25 & 24,3 \\
40 a 49 & 23 & 22,3 \\
50 a 59 & 23 & 22,3 \\
60 anos ou mais & 8 & 7,8 \\
$\quad$ Total & 103 & 100,0 \\
Escolaridade & & \\
Analfabeta & 2 & 1,9 \\
Ensino fundamental incompleto & 19 & 18,4 \\
Ensino fundamental completo & 16 & 15,6 \\
Ensino médio incompleto & 4 & 3,9 \\
Ensino médio completo & 46 & 44,6 \\
Ensino superior incompleto & 4 & 3,9 \\
Ensino superior completo & 12 & 11,7 \\
$\quad$ Total & 103 & 100,00 \\
\hline
\end{tabular}


56 palavras. O núcleo central das representações foi "normal", expressando que esse fator não é um motivo de preocupação para elas. Percebe-se que as mulheres brancas compreendem que a cor branca é a normalidade, é a referência universal. Por isso, as evocações não abordam o privilégio vinculado a tais vivências (25). Ao pesquisar as representações sociais sobre ser lésbica, destacam-se, nas 418 evocações, o pensamento religioso de que Deus fez homem e mulher para ficarem juntos, o preconceito, a ideia de anormalidade e o conceito de família tradicional. Nenhuma mulher se declarou lésbica.

A saúde da mulher foi reconhecida pelas participantes como uma esfera que exige cuidados específicos; essas mulheres apontaram uma grande preocupação com sua saúde reprodutiva, mostrando-se cuidadosas e atentas com os seus exames preventivos, como a mamografia e o Papanicolaou. Vale destacar que elas reconheciam que alguns problemas se devem à sua rotina, que não permite que dediquem mais tempo para si mesmas, o que acaba impossibilitando a prática de exercícios, por exemplo, e faz com que se sintam constantemente cansadas, fisicamente esgotadas e, no geral, sobrecarregadas, devido ao acúmulo de atividades domésticas e cuidado com os filhos, maridos e netos. Mesmo as que trabalhavam fora de casa detinham a maioria ou a totalidade das responsabilidades com o lar e a família. Como manifestou uma participante (E.2), "cuida dos outros e depois de si".

As mulheres apontaram obstáculos nos serviços para os cuidados com a saúde. Segundo elas, os mais graves são: falta de medicamentos, dificuldade de realizar exames e demora para receber os resultados; no caso da saúde mental, dificuldade de conseguir consulta com psicólogo ou psiquiatra. Os médicos cubanos foram elogiados não apenas por acertarem o diagnóstico nas consultas, mas pela alta resolutividade dos tratamentos, recomendações e medicamentos receitados por eles.

Em relação ao cuidado da saúde devido à violência doméstica, as mulheres relataram que as situações de violência ainda não são compreendidas como problemas de saúde. Por isso, a primeira instância, na opinião dessas mulheres, seria a delegacia, sendo que os serviços de saúde só seriam procurados como último recurso, em casos de maior gravidade ou quando houvesse alguma complicação ou lesão decorrente.

\section{A experiência das participantes com os médicos cubanos do PMM}

A tabela 2 mostra a distribuição etária e a escolaridade das 120 mulheres que participaram de grupos focais. As mulheres relataram que, apesar do receio inicial com a chegada dos médicos cubanos, potencializado, em grande medida, pela mídia (26), vivenciaram, por meio das experiências, muitas mudanças positivas na saúde. Apesar do efeito da interseccionalidade na vida delas, consideraram não haver diferenças no atendimento dos médicos do programa pela condição sexual e racial das usuárias.

Não tem diferença nenhuma. Aqui não. No caso de atender, eles atendem toda a mulherada tudo igual. Não tem diferença porque se tivesse, no caso a gente perceberia. (E.1)

Muitas das evocações e relatos fizeram referência ao modelo de atenção humanizado e apontaram que, com a mudança de modelo, houve mudanças na vida delas.
TABELA 2. Idade e escolaridade das usuárias do Programa Mais Médicos que participaram de grupos focais em municípios selecionados, Brasil, 2018

\begin{tabular}{|c|c|c|}
\hline Característica & No. & $\%$ \\
\hline \multicolumn{3}{|l|}{ Idade (anos) } \\
\hline 18 a 29 & 19 & 15,8 \\
\hline 30 a 39 & 23 & 19,2 \\
\hline 40 a 49 & 20 & 16,7 \\
\hline 50 a 59 & 28 & 23,3 \\
\hline 60 ou mais & 30 & 25 \\
\hline Total & 120 & 100 \\
\hline \multicolumn{3}{|l|}{ Escolaridade } \\
\hline Analfabeta & 10 & 8,3 \\
\hline Ensino fundamental incompleto & 50 & 41,7 \\
\hline Ensino fundamental completo & 8 & 6,7 \\
\hline Ensino médio incompleto & 8 & 6,7 \\
\hline Ensino médio completo & 25 & 20,8 \\
\hline Ensino superior incompleto & 7 & 5,8 \\
\hline Ensino superior completo & 8 & 6,7 \\
\hline Sem resposta & 4 & 3,3 \\
\hline Total & 120 & 100 \\
\hline
\end{tabular}

Mas assim, eles mudaram nossa vida sim. Isso nós temos que agradecer. (E.1)

As 441 evocações sobre os serviços de atenção básica mostram que as representações sociais das mulheres sobre o atendimento na UBS e no contexto de ter médicos cubanos atuando foram positivas. As representações sociais sobre ter médicos cubanos no município também foram positivas (430 evocações). Nas representações sobre os médicos brasileiros, as participantes mencionaram um "preconceito de classe" por parte desses médicos em relação a elas (27). Destacaram em relação aos médicos cubanos o atendimento humanizado e não-hierárquico.

Houve consenso entre as participantes quanto à observação de que os médicos cubanos se preocupavam mais com a saúde do que com os ganhos financeiros e não limitavam o trabalho pela quantidade de pacientes atendidos:

\section{Ele não tem aquela preocupação de horário, de quantidade. (E.3)}

Nesse sentido, o fato de as consultas não serem feitas apressadamente e de os médicos escutarem as demandas foi sinalizado como algo que promove o tratamento igualitário e otimiza a experiência clínica:

Antes de ser médico, vocêé uma pessoa, um ser humano (...) O sentimento que a gente tem hoje é que a gente é valorizada. (E.5)

O cuidado durante a consulta e os exames clínicos foram citados como prova de amor ao próximo e como algo que permitia que os médicos cubanos fossem precisos nos tratamentos, diagnosticando doenças que haviam sido investigadas por outros médicos sem terem sido identificadas adequadamente:

Eu estou viva. Eu tenho muito a agradecer a essa UBS porque esse médico, ele tem amor ao próximo. (E.1) 
As mulheres citaram mudanças relacionadas à valorização e à autoestima com a chegada dos médicos:

Quando você aprende a cuidar de você mesma você quer: trabalhar, estudar, e na minha vida eu aprendi que eu me amo, que eu sou bonita (risos) que eu quero viver. (E.1)

\section{A autoestima da gente. A gente fica mais confiante. (E.5)}

As experiências bem-sucedidas com os médicos mudaram a vida das mulheres no sentido de maior autoestima e valorização, temas que constroem empoderamento (12) basicamente em nível individual.

As mulheres expressaram que o tempo de espera diminuiu, que as prioridades ficaram melhor estabelecidas, que a demanda espontânea passou a funcionar, que a rotatividade de médicos acabou e que passou a haver regularidade de visitas domiciliares e atendimento, inclusive nas áreas rurais. Também houve consenso sobre os médicos cubanos se preocuparem em não limitar o atendimento pelo número de pacientes atendidos. $\mathrm{O}$ fato de as participantes conseguirem resolver as questões de saúde mais rapidamente liberou tempo para outras atividades, mas esse "tempo extra" foi pouco usado para elas próprias, sendo majoritariamente dedicado ao cumprimento das tarefas domésticas.

\section{DISCUSSÃO}

Conforme mostrou o presente estudo, o PMM e, dentro dele, a participação de médicos cubanos, trouxeram mudanças positivas para o atendimento de mulheres em quatro municípios brasileiros. Resultados semelhantes foram relatados anteriormente, com evidências de contribuição do PMM para a redução das desigualdades no acesso (28), aumento na satisfação dos usuários (29), melhoria na comunicação (30) e na integralidade das práticas (5) e melhores resultados mensurados pela produção de consultas, encaminhamentos e atendimentos educativos (31). Outros estudos mostraram que os médicos cubanos, especificamente, contribuíram para o fortalecimento da APS (32) e trouxeram ao programa um componente de humanização da atenção, novo para muitos usuários (33).

Sobre o grupo de participantes, destaca-se que, nos municípios escolhidos, não houve diferenças importantes no perfil de idade e escolaridade das usuárias. Sabe-se que o SUS geralmente atende uma população de adultos de baixa renda e baixa escolaridade, pretos e pardos (34). Em relação à questão feminina, para além das dificuldades relacionadas ao gênero, o presente estudo mostrou uma percepção diferente entre negras e brancas, sendo que a cor branca parece ser tomada como referência universal. Por isso, as evocações não abordaram o privilégio vinculado a tais vivências (25), ilustrando o silenciamento e a omissão sobre o racismo por parte da branquitude, a qual dissemina seus privilégios e se beneficia, concreta ou simbolicamente, das desigualdades. Ao pesquisar as representações sociais sobre ser lésbica, as evocações refletiram um forte sentimento de homofobia na cultura dessas mulheres, também presente na cultura brasileira (35).

Houve consenso entre as participantes quanto à observação de que os médicos cubanos se preocupavam mais com a saúde do que com os ganhos financeiros. Outra pesquisa sobre o tema expressou a sensação dos usuários de especulação financeira dos médicos locais e de humanização da atenção dos médicos cubanos (36). Assim, o PMM parece ter contribuído para o fortalecimento da atenção básica, com ganhos para a gestão municipal nos municípios selecionados conforme relatos dos gestores entrevistados, embora persistissem desafios na organização dos serviços, referência e contrarreferência.

O presente estudo apresenta limitações que precisam ser consideradas. Primeiro, a amostra utilizada foi qualitativa, não sendo os resultados generalizáveis para outras situações. Além disso, apenas quatro municípios foram investigados, com entrevistas a um número pequeno de gestores e médicos. Por outro lado, um ponto forte foi a compreensão alcançada, mais aprofundada, acerca do desenvolvimento dos médicos cubanos e das mudanças na vida e saúde de mulheres.

Em conclusão, as mulheres do estudo demonstraram que carregam sobre si a violência de gênero, classe social e, em alguns casos, raça e orientação sexual, eixos de subordinação invisibilizados no cotidiano, inclusive nos serviços de saúde. Entretanto, essa subordinação não foi percebida em relação aos médicos cubanos do PMM, e as mulheres perceberam mudanças positivas na saúde e na própria vida relacionadas ao programa. Destacaram, mais especificamente, mudanças relacionadas a respeito, humanização da atenção e aumento da autoestima, questões relativas à qualidade da atenção e, em menor medida, às práticas de cuidado de sua própria saúde, já que dedicam o tempo de cuidado, fundamentalmente, aos outros.

Finalmente, o PMM parece ter trazido ganhos no empoderamento individual, situação relacionada a uma visão mais ampla de saúde, que colabora para lidar com o racismo, sexismo e homofobia. Deve-se trabalhar para sensibilizar os serviços de saúde sobre as violências invisibilizadas em nível social, como forma de melhorar a saúde das mulheres.

Contribuição dos autores. YC planejou e coordenou a pesquisa e foi responsável pela concepção, redação e revisão do artigo. LLP, PS e IR coletaram e analisaram os dados e participaram da redação/revisão do artigo. CR e GV revisaram o artigo criticamente e participaram da revisão final. Todos os autores aprovaram a versão final.

Conflitos de interesse. Nada declarado pelos autores.

Financiamento. O estudo foi financiado pela Organização Pan-Americana da Saúde (OPAS).

Declaração. As opiniões expressas no manuscrito são de responsabilidade exclusiva dos autores e não refletem necessariamente a opinião ou política da RPSP/PAJPH ou da Organização Pan-Americana da Saúde (OPAS).

\section{REFERÊNCIAS}

1. Campos GWS, Pereira Júnior N. A atenção primária e o Programa Mais Médicos no Sistema Único de Saúde: conquistas e limites. Cienc Saude Coletiva. 2016;21(9):2655-63.
2. Brasil. Medida provisória 621/2013. Disponível em: https:// www2.camara.leg.br/legin/fed/medpro/2013/medidaprovisoria -621-8-julho-2013-776449-norma-pe.html Acessado em abril de 2019. 
3. Brasil. Programa Mais Médicos - dois anos: mais saúde para os brasileiros. Brasília: Ministério da Saúde; 2015. Disponível em: http:/ / dkw4g894e1jim.cloudfront.net/images/PDF/Livro_2_Anos _Mais_Medicos_Ministerio_da_Saude_2015.pdf Acessado em 20 de setembro de 2018.

4. Almeida PF, Giovanella L, Nunan BA. Coordenação dos cuidados em saúde pela atenção primária à saúde e suas implicações para a satisfação dos usuários. Saude Debate. 2012;36(94):375-91.

5. Comes Y, Trindade JS, Pessoa VM, Barreto ICHC, Shimizu HE, Dewes D, et al. A implementação do Programa Mais Médicos e a integralidade nas práticas da Estratégia Saúde da Família. Cienc Saude Colet. 2016;21(9):2729-38.

6. Crenshaw K. Documento para o Encontro de Especialistas em Aspectos da Discriminação Racial Relativos ao Gênero. Rev Estud Fem. 2002;10(1):171-88.

7. Heilborn ML, Araújo L, Barreto A, orgs. Gestão de Políticas Públicas em Gênero e Raça / GPP - GeR: módulo V. Rio de Janeiro: CEPESC; Brasília: Secretaria de Políticas para as Mulheres; 2011.

8. Sousa MO, Sirelli PM. Nem santa, nem pecadora: novas roupagens, velhas dicotomias na coisificação da mulher. Serv Soc Soc. 2018;(132):326-45.

9. Heidari S, Babor TF, Castro P, Tort S, Curno M. Equidade de sexo e gênero na pesquisa: fundamentação das diretrizes SAGER e uso recomendado. Epidemiol Serv Saude. 2017;26(3):665-76.

10. Porto RTS, Bispo Júnior JP, Lima EC. Violência doméstica e sexual no âmbito da Estratégia de Saúde da Família: atuação profissional e barreiras para o enfrentamento. Physis. 2014;24(3):787-807.

11. Carvalho CS, Calderaro F, Souza SJ. O dispositivo "saúde de mulheres lésbicas" : (in)visibilidadee direitos. Rev Psicol Polit. 2013;13(26):111-27.

12. Baquero RVA. Empoderamento: instrumento de emancipação social? - uma discussão conceitual. Rev Debates. 2012;6(1):173.

13. Gohn G. Empoderamento e participação da comunidade em políticas sociais. Saude Soc. 2004;13(2):20-31.

14. Mageste G, Melo M, Ckagnazaroff I. Empoderamento de mulheres: uma proposta de análise para organizações. V Encontro de Estudos Organizacionais da ANPAD - EnEO 2008. Belo Horizonte: ANPAD; 2008. Disponível em: http://www.anpad.org.br/admin/pdf/En EO548.pdf Acessado em abril de 2019.

15. Cyril S, Smith BJ, Renzaho AMN. Systematic review of empowerment measures in health promotion. Health Promot Int. Health Promot Int. 2016;31(4):809-26.

16. Yin R. Estudo de caso: planejamento e métodos. 4a ed. Porto Alegre: Bookman; 2010.

17. Geoff P, Judy P. Key concepts in social research. $1^{\mathrm{a}}$ ed. Londres: Sage Publications Ltd.; 2004

18. Sampieri RH, Collado CF, Lucio PB. Metodología de la investigación. $6^{a}$ ed. México: McGraw-Hill/Interamericana Editores, S.A.; 2017. Disponível em: http:/ /observatorio.epacartagena.gov.co/wp -content/uploads/2017/08/metodologia-de-la-investigacion-sexta -edicion.compressed.pdf Acessado em 2 de outubro de 2018.

19. Werneck J. Racismo institucional e saúde da população negra. Saude Soc. 2016;25(3):535-49.

20. Jose B, Fontanella B. Amostragem em pesquisas qualitativas : proposta de procedimentos para constatar saturação teórica. Cad Saude Publica. 2011;27(2):389-94.

21. Wachelke J, Wolter R. Criteria related to the realization and reporting of prototypical analysis for social representations. Psic Teor Pesq. 2011;27(4):521-6.
22. Bardin L. Análise de conteúdo. Lisboa: Edições 70; 2011.

23. Bericat E. La integración de los métodos cuantitativo y cualitativo en la investigación social. Significado y medida. Barcelona: Editora Ariel; 1998.

24. Narvaz MG, Koller SH. Famílias e patriarcado: da prescrição normativa à subversão criativa. Psicol Soc. 2006;18(1):49-55.

25. Carneiro S. Enegrecer o feminismo: a situação da mulher negra na América Latina a partir de uma perspectiva de gênero. Em: Ashoka Empreendimentos Sociais \& Takano Cidadania, orgs. Racismos contemporâneos. Rio de Janeiro: Takano Editora; 2003. Pp. 49-58.

26. Ramos R. Programa Mais Médicos: aversão e preconceito. Uma análise na perspectiva do pensamento de Frantz Fanon. Csonline. 2017;(24):1-17.

27. Mendonça MLM, Jordão JVP. Nojo de pobre : representações do popular e preconceito de classe. Contemporanea. 2014;1(23):1-18

28. Oliveira JPA, Sanchez MN, Santos LMP. O Programa Mais Médicos: provimento de médicos em municípios brasileiros prioritários entre 2013 e 2014. Cienc Saude Colet. 2016;21(9):2719-27.

29. Comes Y, Trindade J de S, Shimizu HE, Hamann EM, Bargioni F, Ramirez L, et al. Avaliação da satisfação dos usuários e da responsividade dos serviços em municípios inscritos no Programa Mais Médicos. Cienc Saude Colet. 2016;21(9):2749-59.

30. Silva TRB da, Silva J do V e, Pontes AGV, Cunha ATR da. Percepção de usuários sobre o Programa Mais Médicos no município de Mossoró, Brasil. Cienc Saude Colet. 2016;21(9):2861-9.

31. Lima RT de S, Fernandes TG, Balieiro AA da S, Costa F dos S, Schramm JM de A, Schweickardt JC, et al. A Atenção Básica no Brasil e o Programa Mais Médicos: uma análise de indicadores de produção. Cienc Saude Colet. 2016;21(9):2685-96.

32. Organização Pan-Americana da Saúde (OPAS). Informe estratégico sobre o Programa Mais Médicos e saúde universal. Brasília, DF: OPAS; 2018. Disponível em: http://iris.paho.org/xmlui/bitstream $/$ handle $/ 123456789 / 49245 /$ OPASBRA18013-por.pdf?sequence $=$ 1\&isAllowed =y Acessado em abril de 2019.

33. Comes Y, Díaz-Bermúdez XP, Pereira LL, Oliveira FP, Caballero González JE, Shimizu HE, et al. Humanismo en la práctica de médicos cooperantes cubanos en Brasil: narrativas de equipos de atención básica. Rev Panam Salud Publica. 2017;41:e130.

34. Sampaio de Almeida Ribeiro MC, Barradas Barata R, Furquim de Almeida M, Pereira da Silva Z. Perfil sociodemográfico e padrão de utilização de serviços de saúde para usuários e não-usuários do SUS - PNAD. Cienc Saude Colet. 2006;11(4):1011-22.

35. Albuquerque GA, Garcia C de L, Alves MJH, Queiroz CMHT de, Adami F. Homossexualidade e o direito à saúde: um desafio para as políticas públicas de saúde no Brasil. Saude Debate. 2013;37(98):516-24.

36. Melo CDF, Baião DC, Costa MC. A percepção dos usuários cearenses sobre o Programa Mais Médicos. Gestao Soc. 2016;10(26): 1302.

Manuscrito recebido em 26 de dezembro de 2018. Aceito em versão revisada em 20 de março de 2019 


\section{Women's health and empowerment: a case study of the More Doctors Program in municipalities with Cuban physicians}

ABSTRACT Objective. To describe the representations of being a woman by users of the More Doctors Program (Programa Mais Médicos, PMM) in Brazil, exploring the perspectives of gender and race, and the changes produced by PMM in terms of empowerment and health care.

Methods. This is a descriptive, cross-sectional study. The field work was performed using semi-structured interviews, with application of an evocative word technique and focal groups in municipalities with Cuban physicians, with nominal selection of municipalities and intentional selection of participants. The size of the sample was defined in the field based on saturation. The data were analyzed by content and prototypical analyses.

Results. Primary health care coverage was strengthened by the PMM, according to the four municipal health secretaries interviewed. Participants (103 in the evocative technique and 120 from focal groups) reported changes in the model of care, which became more humanized, with impact on their perception of health care services, medical consultations, and physicians, on the image they had of themselves and, to a lesser extent, on their health care practices.

Conclusions. PMM produced individual empowerment gains for study participants, with potentially positive impacts on health care behaviors.

Keywords

National health programs; women's rights; comprehensive health care; public nondiscriminatory policies; primary health care; Brazil.

\section{Salud y empoderamiento de las mujeres: estudio de caso del programa Mais Médicos en municipios con presencia de médicos cubanos}

RESUMEN

Objetivo. Describir las representaciones de la condición de ser mujer hechas por las usuarias del programa Mais Médicos, con una perspectiva de género y raza, y los cambios producidos por este programa en materia de empoderamiento y cuidado de la salud.

Métodos. Se trata de un estudio de caso descriptivo y transversal. El trabajo de campo se realizó mediante entrevistas semiestructuradas, con aplicación de una técnica evocadora de asociación de palabras y grupos focales en municipios con presencia de médicos cubanos, con muestras de tipo nominal para la selección de los municipios y de tipo intencional para la selección de las participantes. El tamaño de las muestras se definió sobre el terreno con base en la técnica de la saturación teórica. Los datos se sometieron a análisis prototípico y de contenido.

Resultados. Los aportes del programa fortalecieron la cobertura de la atención básica, según lo expresado por los cuatro gestores entrevistados. Las mujeres (103 de las entrevistadas con la técnica evocadora y 120 de los grupos focales) relataron cambios en los modelos de atención que hicieron que la atención se torne más humanizada y que incidieron en su percepción de los servicios de salud, las consultas médicas, los médicos, la imagen de sí mismas y, en menor grado, las prácticas de cuidado de la salud.

Conclusiones. El programa Mais Médicos implicó adelantos en materia de empoderamiento individual de las mujeres, con repercusiones potencialmente favorables en los patrones de comportamiento relacionados con la salud.

Palabras clave Programas nacionales de salud; derechos de la mujer; atención integral de salud; políticas públicas antidiscriminatorias; Brasil. 